\title{
Impact of body mass index on quality of life after distal gastrectomy for gastric cancer
}

\author{
Ki Bum Park, Byunghyuk Yu, Ji Yeon Park, Oh Kyoung Kwon, Wansik Yu \\ Gastric Cancer Center, Kyungpook National University Chilgok Hospital, Daegu, Korea
}

\begin{abstract}
Purpose: Few studies have evaluated changes in quality of life (QoL) in relation to changes in body mass index (BMI) after gastrectomy. This study aimed to evaluate the impact of postoperative changes in BMI on QoL after distal gastrectomy in gastric cancer patients.

Methods: QoL data from the European Organization for the Research and Treatment of Cancer (EORTC) gathered via the QLQ-C30 and QLQ-STO22 questionnaires were obtained from 1,036 patients preoperatively and at 1 year postoperatively. The patients were divided into 2 groups: group 1 - decreased postoperative BMI and group 2 - unchanged or increased postoperative BMI.

Results: There were 577 patients in group 1 and 459 in group 2. According to global health status and functional scales, emotional functioning $(P=0.035)$ was significantly worse in group 1 than in group 2 at 1 year postoperatively. Furthermore, there were significant decreases in QoL symptom scale scores, including fatigue $(P=0.016)$, nausea and vomiting $(P=0.002)$, and appetite loss $(P=0.001)$ scores, in group 1 compared with group 2. Regarding QLQ-STO22, reflux symptoms $(P=0.020)$, anxiety $(P=0.003)$, and body image $(P=0.003)$ were significantly worse in group 1 than in group 2 at 1 year after surgery. Conclusion: BMI changes after distal gastrectomy influence QoL. Focus on controlling gastrointestinal symptoms and providing psychological support is essential in patients with decreased BMI after surgery. Patients should be offered follow-up care to assist them in maintaining BMI, for example, through dietary-behavior modifications and via intensive nutritional support, to prevent QoL deterioration after distal gastrectomy.
\end{abstract}

[Ann Surg Treat Res 2019;96(5):250-258]

Key Words: Quality of life, Body mass index, Gastrectomy, Stomach neoplasm

\section{INTRODUCTION}

Surgery is the only curative treatment for gastric cancer with the ultimate goal of improved survival. Additional outcomes reflecting the effectiveness of surgical treatment for gastric cancer include safety measures such as short-term morbidity and mortality and functional outcomes including postoperative performance status. Assessing quality of life (QoL) after gastrectomy can be an objective indicator when these items are evaluated [1].

Patients undergoing gastrectomy for gastric cancer may experience reduced QoL postoperatively, which tends to be lowest during the first year after gastrectomy [2,3]. Another potential problem during this period is nutritional deficiency. Changes in body composition, including weight loss, are not preventable after gastrectomy and usually persist until 1 year postoperatively. Additionally, most patients do not recover to their preoperative body weight after gastrectomy [4,5]. These 2 changes are closely related to each other; however, little is known about the connection between changes in body weight and QoL after gastrectomy for gastric cancer. Thus, investigation of any relationship between QoL and weight change in patients
Received June 26, 2018, Revised December 3, 2018,

Accepted December 21, 2018

Corresponding Author: Wansik Yu

Gastric Cancer Center, Kyungpook National University Chilgok Hospital,

807 Hoguk-ro, Buk-gu, Daegu 41404, Korea

Tel: +82-53-200-2700, Fax: +82-53-200-2027

E-mail: wyu@knu.ac.kr

ORCID code: https://orcid.org/0000-0002-5067-8501
Copyright (C) 2019, the Korean Surgical Society

(c) Annals of Surgical Treatment and Research is an Open Access Journal. All articles are distributed under the terms of the Creative Commons Attribution NonCommercial License (http://creativecommons.org/licenses/by-nc/4.0/) which permits unrestricted non-commercial use, distribution, and reproduction in any medium, provided the original work is properly cited. 
who undergo distal gastrectomy is important.

In recent years, interest in QoL after surgery has increased, owing largely to the fact that QoL is directly related to patient outcomes after surgery [1]. Although many clinicians are aware of the importance of QoL assessment and believe that QoL is a critical clinical endpoint, the clinical measurement of QoL is infrequent [6]. However, since the diagnostic tools for QoL assessment have recently been improved and validated, their use is expanding into clinical settings. Among these diverse tools, the European Organization for Research and Treatment of Cancer (EORTC) Quality of Life Questionnaire (QLQ)-C30 with the gastric cancer-specific module (QLQ-STO22) has been demonstrated to have good reliability and validity $[7,8]$.

QoL changes after gastrectomy in patients with gastric cancer using the EORTC QLQ-C30 and QLQ-STO22 have been previously reported. However, most of the previous reports compared QoL changes based on the extent of surgical procedures or QoL change flow throughout the postoperative survival period [9-12]. Moreover, few studies evaluated QoL changes with respect to changes in body mass index (BMI) after gastrectomy [13]. Therefore, we investigated QoL changes during the first year after surgery with respect to BMI shifts, with the goal of understanding specific QoL deterioration and providing tailored medical intervention by revealing additional factors that could improve the QoL of patients undergoing a distal gastrectomy.

\section{METHODS}

\section{Patients}

Patients with gastric cancer who underwent curative distal gastrectomy between January 2011 and December 2014 at the Kyungpook National University Chilgok Hospital were enrolled. We excluded patients who experienced a recurrence within 1 year after surgery, received neoadjuvant chemotherapy, or died of other causes. Ultimately, 1,036 patients who completed the entire series of QoL assessments during the first year were analyzed. All procedures followed were in accordance with the ethical standards of the responsible committee on human experimentation (institutional and national) and with the Helsinki Declaration of 1964 and later versions. The Institutional Review Board of Kyungpook National University Chilgok Hospital approved this study (approval number: 201707-018). Written informed consent was waived by the IRB.

BMI was calculated as body weight/height ${ }^{2}\left(\mathrm{~kg} / \mathrm{m}^{2}\right)$, and based on these values, the patients were classified as underweight $\left(<18.50 \mathrm{~kg} / \mathrm{m}^{2}\right)$, normal $\left(18.50-22.99 \mathrm{~kg} / \mathrm{m}^{2}\right)$, overweight $(23.00$ $\left.24.99 \mathrm{~kg} / \mathrm{m}^{2}\right)$, and obese $\left(\geq 25.00 \mathrm{~kg} / \mathrm{m}^{2}\right.$ ) (Fig. 1) [14]. Patients were divided into 2 groups based on their BMI shifts: group 1 $(n=577)$ included patients whose BMIs were in lower ranges than their preoperative classifications, while group $2(n=459)$ comprised patients with their BMI ranges maintained $(\mathrm{n}=$ 434) or shifted to a higher range than their preoperative BMI $(\mathrm{n}=25)$. Moreover, patients were divided into 3 groups for subgroup analysis: Subgroup $1(\mathrm{n}=101)$ included patients with BMI change from normal to underweight range, subgroup 2 (n = 337) included patients with BMI change from overweight and obese ranges to normal range, and subgroup $3(n=434)$ included patients with BMI ranges maintained after surgery.

\section{Surgery}

Curative distal gastrectomy and D2 lymph node dissection including total omentectomy were performed for advanced gastric cancer, whereas D1+ lymph node dissection including partial omentectomy was performed for early gastric cancer. Billroth I reconstruction procedures (BI anastomosis) were performed extracorporeally using a circular stapler for open distal gastrectomy and laparoscopy-assisted distal gastrectomy [15]. In totally laparoscopic distal gastrectomy, BI anastomosis was performed using the Delta anastomosis method [16]. After surgery, patients were managed based on a clinical protocol that included drinking water intake on the third postoperative day, followed by liquid diet initiation on the fourth postoperative day and soft diet consumption on the fifth postoperative day. Patients were planned for discharge on the sixth postoperative day.

\section{QoL assessments}

The Korean versions of the EORTC QLQ-C30 and EORTC QLQ-STO22 [17] were used to assess the QoL of patients. Patients were asked to fill the QLQ-C30 and QLQ-STO22 questionnaires twice: preoperatively and at 1 year after surgery. The QoL assessment included patients who responded to all items of the questionnaire by themselves. The preoperative QoL

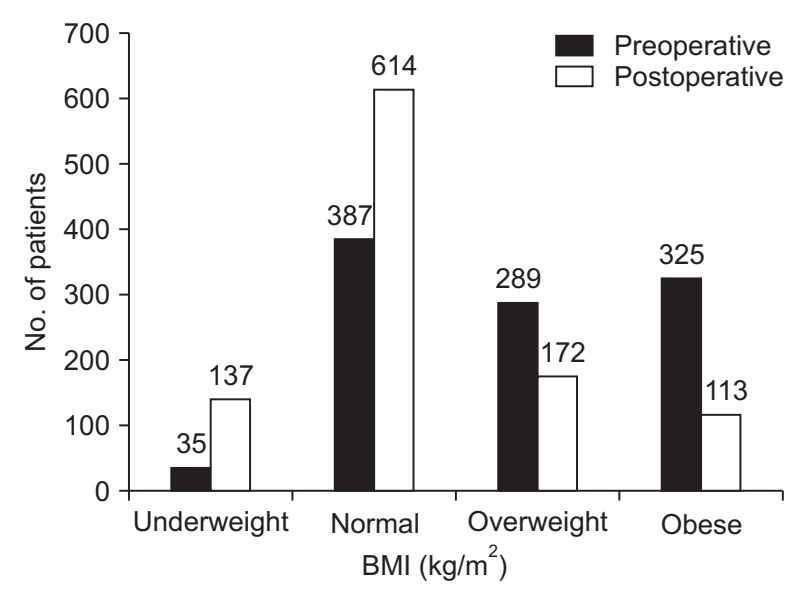

Fig. 1. Body mass index shift after distal gastrectomy. Underweight $\left(<18.50 \mathrm{~kg} / \mathrm{m}^{2}\right)$, normal $\left(18.50-22.99 \mathrm{~kg} / \mathrm{m}^{2}\right)$, overweight $\left(23.00-24.99 \mathrm{~kg} / \mathrm{m}^{2}\right)$, and obese $\left(\geq 25.00 \mathrm{~kg} / \mathrm{m}^{2}\right)$. 
assessment was performed when the patients were hospitalized for surgery and the postoperative QoL assessment was performed at the outpatient department during regular followup. If questionnaires were returned with missing individual items, patients were required to fill the missing individual items. The raw scores were linearly transformed into scores ranging from 0 to 100 according to the manual provided by the EORTC. For global health status/QoL and functional scales, a higher score can be interpreted as a high QoL and better functioning but in symptoms scales and items, it reflects more symptoms/problems. For EORTC QLQ-STO22, a higher score can be interpreted as a low QoL [18].

\section{Statistical analysis}

Differences in baseline characteristics of patients between the 2 groups or 3 subgroups were analyzed with the chi-square test for categorical variables and Student t-test for continuous variables. The mean score of QoL changes between preoperative and postoperative values was analyzed with the paired t-test.

Linear mixed models with first-degree autoregressive covariance matrix were used to assess how the surgery affected the changes in QoL between the groups and over time. The baseline characteristics of patients were included as covariates if there was a significant difference. A P-value of $<0.05$ was considered statistically significant. All statistical analyses were conducted using IBM SPSS Statistics ver. 22.0 (IBM Co., Armonk, NY, USA).

\section{RESULTS}

\section{Patients' characteristics}

Table 1 shows the clinicopathological characteristics of the patients. There were no statistical differences in age, sex, type of surgery, coresection, comprehensive complication index, adjuvant chemotherapy, pathological stage, previous surgical history, and previous cancer history between groups 1 and 2. However, statistically significant differences were found between groups in terms of length of hospital stay and comorbidities.

\section{Changes in QoL after surgery}

The mean QoL scores preoperatively and 1 year after surgery for all patients are shown in Fig. 2. The global health status/ QoL was significantly improved after surgery. In functional scales, physical functioning, role functioning, and cognitive functioning were significantly worse; however, emotional functioning was significantly better 1 year postoperatively. In symptom scales, fatigue, nausea and vomiting, and diarrhea demonstrated significant worsening 1 year postoperatively. For EORTC-STO 22, most items except pain and reflux symptoms scale showed significant worsening 1 year postoperatively.
We compared the mean QoL score changes between the groups over time (Fig. 3). Global health status and emotional functioning increased in both groups but the degree of improvement in emotional functioning was better in group 2 than in group 1 at 1 year postoperatively $(P=0.035)$. Functional scales other than emotional functioning were decreased in both groups after surgery, but not significantly. Gastrointestinal symptoms such as fatigue $(P=0.016)$, nausea and vomiting $(P=0.002)$, and appetite loss $(P=0.001)$ decreased more significantly in group 1 than in group 2. For QLQ-STO22 scales and items in both groups, reflux symptoms $(P=0.020)$, anxiety

Table 1. Characteristics of patients between groups 1 and 2

\begin{tabular}{|c|c|c|c|}
\hline Characteristic & $\begin{array}{l}\text { Group } 1 \\
(\mathrm{n}=577)\end{array}$ & $\begin{array}{l}\text { Group } 2 \\
(\mathrm{n}=459)\end{array}$ & P-value \\
\hline Age (yr) & $61.3 \pm 11.8$ & $59.9 \pm 11.6$ & 0.058 \\
\hline Sex & & & 0.071 \\
\hline Female & $235(40.7)$ & $161(35.1)$ & \\
\hline Male & $342(59.3)$ & $298(64.9)$ & \\
\hline Comorbidity & & & 0.001 \\
\hline Yes & $271(47.0)$ & $158(35.1)$ & \\
\hline No & $306(53.0)$ & $301(65.6)$ & \\
\hline Previous operation history & & & 0.783 \\
\hline Yes & $30(5.2)$ & $26(5.7)$ & \\
\hline No & $547(94.8)$ & $433(94.3)$ & \\
\hline Previous cancer history & & & 0.865 \\
\hline Yes & $20(3.5)$ & $15(3.3)$ & \\
\hline No & $557(96.5)$ & $444(96.7)$ & \\
\hline Type of surgery & & & 0.149 \\
\hline $\mathrm{BI}$ & $534(92.6)$ & $432(94.1)$ & \\
\hline BII & $37(6.4)$ & $26(5.7)$ & \\
\hline RY-GJ & $6(1.0)$ & $1(0.2)$ & \\
\hline Coresection & & & 0.441 \\
\hline Yes & $59(10.2)$ & $34(7.4)$ & \\
\hline No & $518(89.9)$ & $425(92.6)$ & \\
\hline Hospital stay (day) & $11.1 \pm 9.4$ & $10.1 \pm 5.1$ & 0.028 \\
\hline $\mathrm{CCl}$ & $1.8 \pm 6.8$ & $1.1 \pm 5.8$ & 0.110 \\
\hline Stage & & & 0.303 \\
\hline IA & $419(72.6)$ & $316(68.8)$ & \\
\hline IB & $61(10.6)$ & $41(8.9)$ & \\
\hline IIA & 34 (5.9) & $37(8.1)$ & \\
\hline IIB & $18(3.1)$ & $24(5.2)$ & \\
\hline IIIA & $15(2.6)$ & $18(3.9)$ & \\
\hline IIIB & $19(3.3)$ & $15(3.3)$ & \\
\hline IIIC & $11(1.9)$ & $8(1.7)$ & \\
\hline Adjuvant chemotherapy & & & 0.145 \\
\hline Yes & $60(10.4)$ & $62(13.5)$ & \\
\hline No & 517 (89.6) & $397(86.5)$ & \\
\hline
\end{tabular}

Values are presented as mean \pm standard deviation or number (\%).

Stage grouping by 7 th edition of the American Joint Committee on Cancer classification.

Group 1, decreased postoperative BMl; group 2, unchanged or increased postoperative BMI; RY-GJ, Roux-en Y gastrojejunostomy; $\mathrm{CCl}$, comprehensive complication index; $\mathrm{BMI}$, body mass index. 

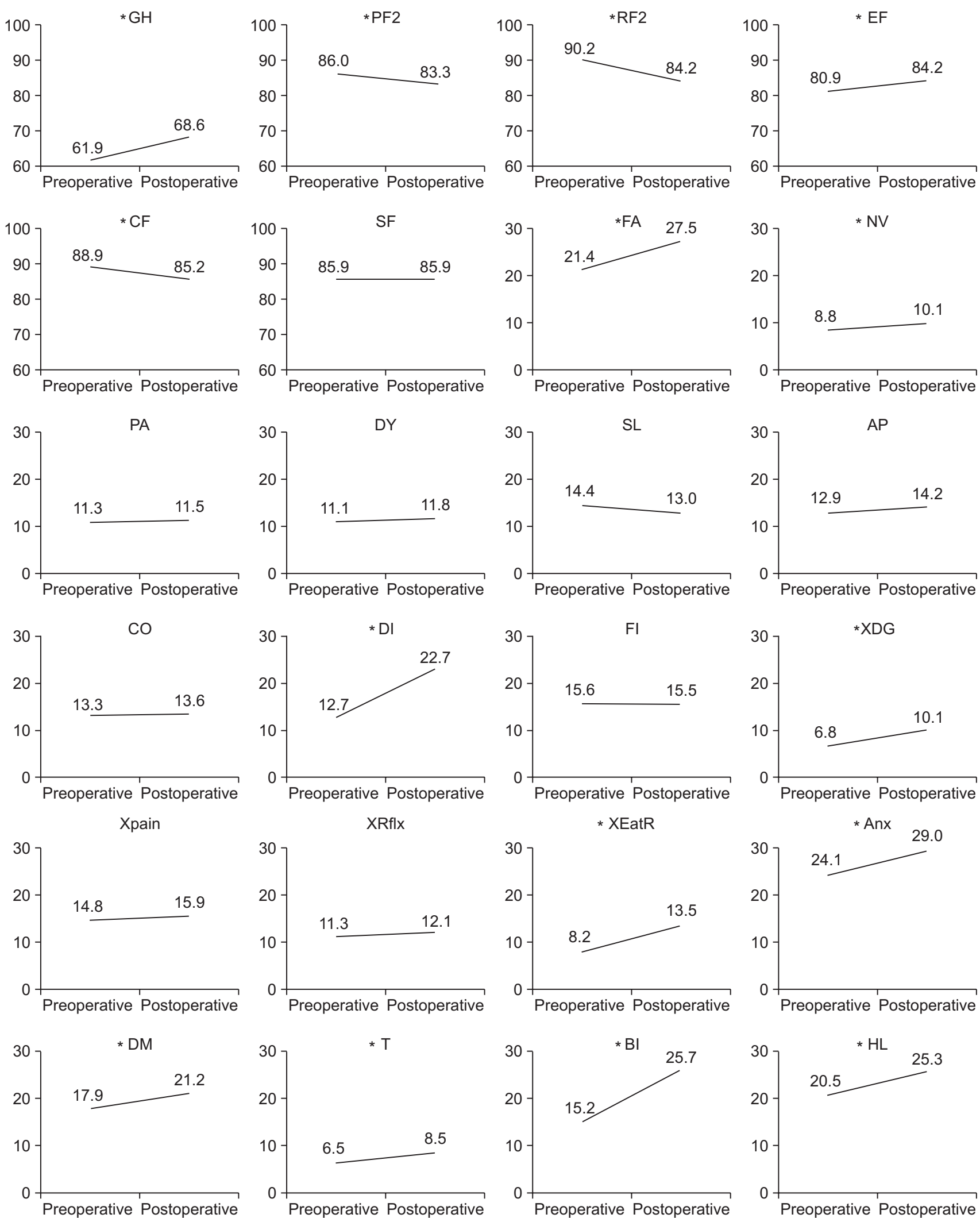

Fig. 2. Mean score of quality of life preoperatively and postoperatively 1 year after distal gastrectomy. GH, global health status; PF2, physical functioning; RF2, role functioning; EF, emotional functioning; CF, cognitive functioning; SF, social functioning; FA, fatigue; NV, nausea and vomiting; PA, pain; DY, dyspnea; SL, insomnia; AP, appetite loss; CO, constipation; DI, diarrhea; FI, financial difficulties; XDG, dysphagia; Xpain, pain; XRflx, reflux symptoms; XEatR, eating restrictions; Anx, anxiety; DM, having a dry mouth; $\mathrm{T}$, taste; $\mathrm{BI}$, body image; $\mathrm{HL}$, hair loss. ${ }^{*} \mathrm{P}<0.05$. 
$(P=0.001)$, and body image $(P=0.003)$ were associated with a significantly worse QoL in group 1 than in group 2.

Table 2 shows the clinicopathological characteristics of
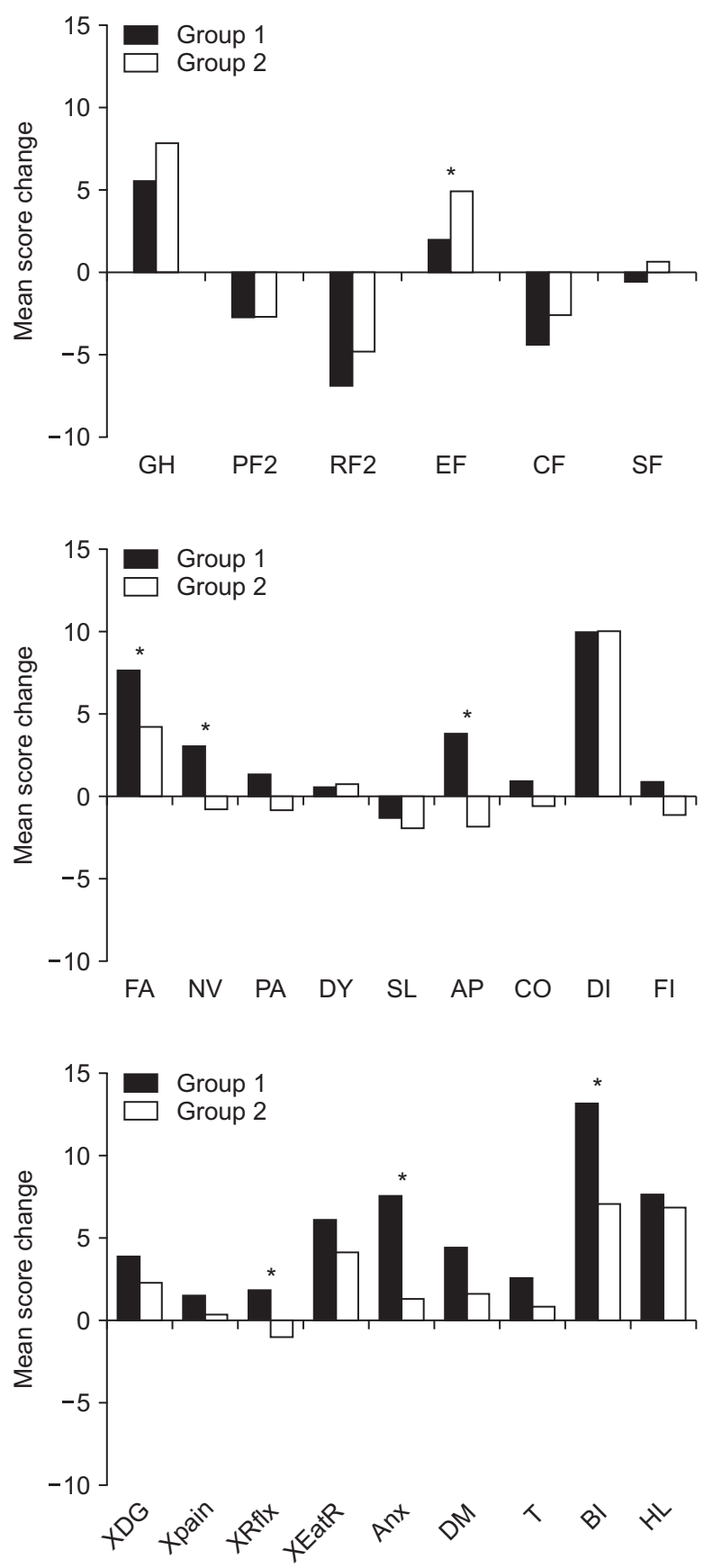

Fig. 3. Changes in quality of life according to body mass index shifts 1 year after distal gastrectomy between groups 1 and 2. GH, global health status; PF2, physical functioning; RF2, role functioning; $E F$, emotional functioning; $C F$, cognitive functioning; SF, social functioning; FA, fatigue; $N V$, nausea and vomiting; PA, pain; DY, dyspnea; SL, insomnia; AP, appetite loss; CO, constipation; DI, diarrhea; FI, financial difficulties; XDG, dysphagia; Xpain, pain; XRflx, reflux symptoms; XEatR, eating restrictions; Anx, anxiety; DM, having a dry mouth; $T$, taste; $\mathrm{BI}$, body image; $\mathrm{HL}$, hair loss. ${ }^{*} \mathrm{P}<0.05$. subgroup 1 and 2. Sex, comorbidity, and pathological stage were significantly different between subgroups. All the QoL categories showed no significant differences among the subgroups 1 year postoperatively (Fig. 4).

Table 3 shows the clinicopathological characteristics of subgroup 2 and 3. Only comorbidity showed a significant difference between subgroups. In the global health status/QoL and functional scales, no significant differences were noted 1 year postoperatively. Regarding gastrointestinal symptoms

Table 2. Characteristics of patients between subgroups 1 and 2

\begin{tabular}{|c|c|c|c|}
\hline Characteristic & $\begin{array}{l}\text { Subgroup } 1 \\
\qquad(\mathrm{n}=101)\end{array}$ & $\begin{array}{l}\text { Subgroup } 2 \\
(\mathrm{n}=337)\end{array}$ & P-value \\
\hline Age (yr) & $61.6 \pm 13.6$ & $61.5 \pm 11.6$ & 0.922 \\
\hline Sex & & & 0.008 \\
\hline Female & $53(52.5)$ & $126(37.4)$ & \\
\hline Male & $48(47.5)$ & $211(62.6)$ & \\
\hline Comorbidity & & & 0.001 \\
\hline Yes & $31(30.7)$ & $163(48.4)$ & \\
\hline No & $70(69.3)$ & $174(51.6)$ & \\
\hline Previous operation history & & & 0.618 \\
\hline Yes & $4(4.0)$ & $19(5.6)$ & \\
\hline No & $97(96.0)$ & $318(94.4)$ & \\
\hline Previous cancer history & & & 0.085 \\
\hline Yes & $0(0)$ & $13(3.9)$ & \\
\hline No & $101(100)$ & $324(96.1)$ & \\
\hline Type of surgery & & & 0.138 \\
\hline $\mathrm{BI}$ & $97(96.0)$ & $311(92.3)$ & \\
\hline BII & $4(4.0)$ & $23(6.9)$ & \\
\hline RY-GJ & $0(0)$ & $3(0.8)$ & \\
\hline Co-resection & & & 0.850 \\
\hline Yes & $9(8.9)$ & $34(10.1)$ & \\
\hline No & $92(91.1)$ & $303(89.9)$ & \\
\hline Hospital stay (day) & $10.2 \pm 6.0$ & $11.3 \pm 10.7$ & 0.317 \\
\hline $\mathrm{CCl}$ & $2.2 \pm 8.0$ & $1.5 \pm 6.2$ & 0.366 \\
\hline Stage & & & 0.039 \\
\hline IA & $61(60.4)$ & $251(74.5)$ & \\
\hline IB & $16(15.8)$ & $30(8.9)$ & \\
\hline IIA & $11(10.9)$ & $17(5.0)$ & \\
\hline IIB & $2(2.0)$ & $13(3.9)$ & \\
\hline IIIA & $5(5.0)$ & $7(2.1)$ & \\
\hline IIIB & $4(4.0)$ & $11(3.3)$ & \\
\hline IIIC & $2(2.0)$ & $8(2.4)$ & \\
\hline Adjuvant chemotherapy & & & 0.459 \\
\hline Yes & $13(12.9)$ & $33(9.8)$ & \\
\hline No & $88(87.1)$ & $304(90.2)$ & \\
\hline
\end{tabular}

Values are presented as mean \pm standard deviation or number (\%).

Stage grouping by 7 th edition of the American Joint Committee on Cancer classification.

Subgroup 1, patients with BMI change from normal BMI ranges to underweight BMI ranges; subgroup 2, patients with BMI change from overweight and obese ranges to normal $\mathrm{BMI}$ ranges; RY-G), Roux-en Y gastrojejunostomy; CCl, comprehensive complication index; $\mathrm{BMI}$, body mass index. 

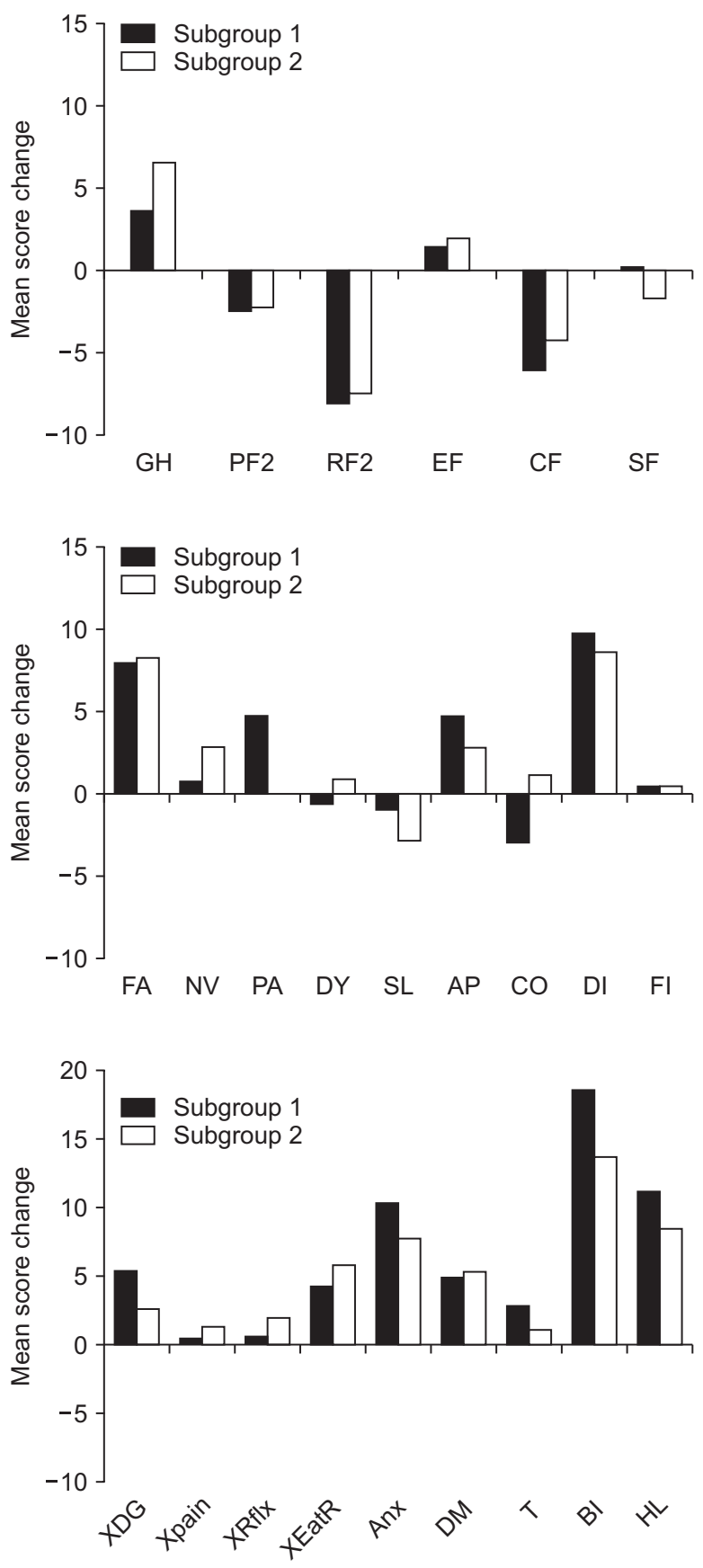

Fig. 4. Changes in quality of life between subgroups 1 and 2. GH, global health status; PF2, physical functioning; RF2, role functioning; $\mathrm{EF}$, emotional functioning; $\mathrm{CF}$, cognitive functioning; SF, social functioning; FA, fatigue; NV, nausea and vomiting; PA, pain; DY, dyspnea; $S L$, insomnia; $A P$, appetite loss; CO, constipation; $\mathrm{DI}$, diarrhea; $\mathrm{FI}$, financial difficulties; XDG, dysphagia; Xpain, pain; XRflx, reflux symptoms; XEatR, eating restrictions; Anx, anxiety; DM, having a dry mouth; T, taste; $\mathrm{BI}$, body image; $\mathrm{HL}$, hair loss.

and QLQ-STO22, fatigue ( $\mathrm{P}=0.032)$, nausea and vomiting $(P=0.035)$, anxiety $(P=0.001)$, and body image $(P=0.012)$ showed significantly better QoL scores in Subgroup 3 at 1 year postoperatively (Fig. 5).
Table 3. Characteristics of patients between subgroups 2 and 3

\begin{tabular}{|c|c|c|c|}
\hline Characteristic & $\begin{array}{l}\text { Subgroup } 2 \\
(\mathrm{n}=337)\end{array}$ & $\begin{array}{c}\text { Subgroup } 3 \\
(\mathrm{n}=434)\end{array}$ & P-value \\
\hline Age (yr) & $61.5 \pm 11.6$ & $59.8 \pm 11.7$ & 0.052 \\
\hline Sex & & & 0.547 \\
\hline Female & $126(37.4)$ & $153(35.3)$ & \\
\hline Male & $211(62.6)$ & $281(64.7)$ & \\
\hline Comorbidity & & & 0.001 \\
\hline Yes & $163(48.4)$ & $151(34.8)$ & \\
\hline No & $174(51.6)$ & $283(65.2)$ & \\
\hline Previous operation history & & & $>0.999$ \\
\hline Yes & $19(5.6)$ & $25(5.8)$ & \\
\hline No & $318(94.4)$ & $409(94.2)$ & \\
\hline Previous cancer history & & & 0.550 \\
\hline Yes & $13(3.9)$ & $13(3.0)$ & \\
\hline No & $324(96.1)$ & $421(97.0)$ & \\
\hline Type of surgery & & & 0.303 \\
\hline $\mathrm{BI}$ & $311(92.3)$ & $408(94.0)$ & \\
\hline BII & $23(6.8)$ & $25(5.8)$ & \\
\hline RY-GJ & $3(0.9)$ & $1(0.2)$ & \\
\hline Coresection & & & 0.306 \\
\hline Yes & $34(10.1)$ & $34(7.8)$ & \\
\hline No & $303(89.9)$ & $400(92.2)$ & \\
\hline Hospital stay (days) & $11.3 \pm 10.7$ & $10.2 \pm 5.3$ & 0.085 \\
\hline $\mathrm{CCl}$ & $1.5 \pm 6.2$ & $1.2 \pm 5.9$ & 0.428 \\
\hline Stage & & & 0.394 \\
\hline IA & $251(74.5)$ & $303(69.8)$ & \\
\hline IB & $30(8.9)$ & $37(8.5)$ & \\
\hline IIA & $17(5.0)$ & $33(7.6)$ & \\
\hline IIB & $13(3.9)$ & $24(5.5)$ & \\
\hline IIIA & $7(2.1)$ & $17(3.9)$ & \\
\hline IIIB & $11(3.3)$ & $13(3.0)$ & \\
\hline IIIC & $8(2.4)$ & $7(1.6)$ & \\
\hline Adjuvant chemotherapy & & & 0.175 \\
\hline Yes & $33(9.8)$ & $57(13.1)$ & \\
\hline No & $304(90.2)$ & 377 (86.9) & \\
\hline
\end{tabular}

Values are presented as mean \pm standard deviation or number (\%).

Stage grouping by 7 th edition of the American Joint Committee on Cancer classification.

Subgroup 2, patients with BMI change from overweight and obese ranges to normal BMI ranges; subgroup 3, patients who had maintained their BMI ranges after surgery; RY-GJ, Roux-en $Y$ gastrojejunostomy; $\mathrm{CCl}$, comprehensive complication index; $\mathrm{BMI}$, body mass index.

\section{DISCUSSION}

Patients undergoing gastrectomy experience various body changes, including changes in body composition, digestive and metabolic disorders, and psychological problems, with most of these changes occurring within the first postoperative year. Objective measures for assessing these changes include blood testing, imaging studies, and body composition analysis, while the QoL questionnaire is a subjective indicator. Although QoL 

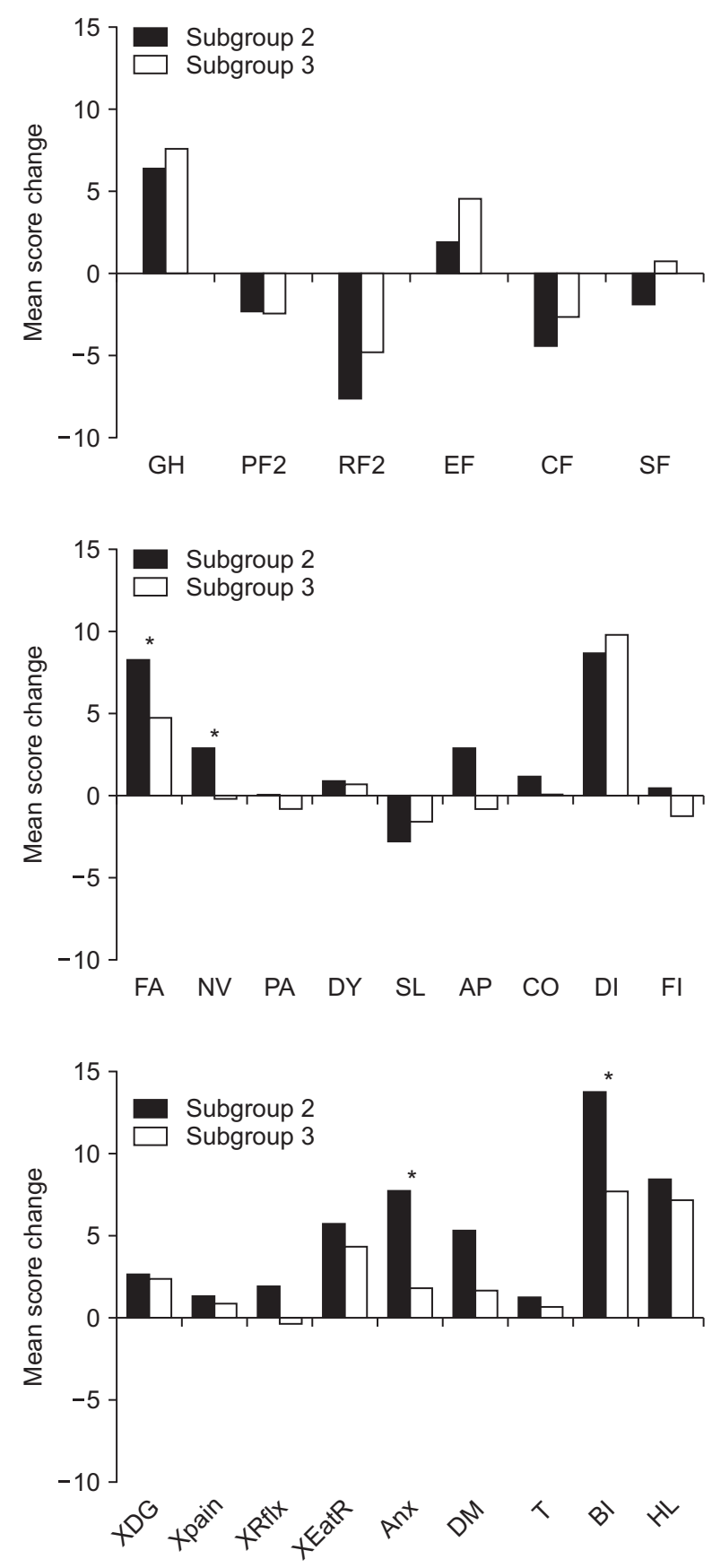

Fig. 5. Changes in quality of life between subgroups 2 and 3. $\mathrm{GH}$, global health status; PF2, physical functioning; RF2, role functioning; $\mathrm{EF}$, emotional functioning; $\mathrm{CF}$, cognitive functioning; SF, social functioning; FA, fatigue; NV, nausea and vomiting; PA, pain; DY, dyspnea; $S L$, insomnia; $A P$, appetite loss; CO, constipation; DI, diarrhea; FI, financial difficulties; XDG, dysphagia; Xpain, pain; XRflx, reflux symptoms; XEatR, eating restrictions; Anx, anxiety; DM, having a dry mouth; T, taste; $\mathrm{Bl}$, body image; $\mathrm{HL}$, hair loss. $* P<0.05$.

assessment is subjective, it has become increasingly important for oncological outcomes and is no longer a soft measurement in assessing surgical outcomes [19]. Generally, patients undergoing distal gastrectomy are expected to experience a better QoL than those undergoing total gastrectomy. Although the former patients may have better QoL, impaired nutrition due to a decrease in stomach volume is inevitable and leads to significant weight loss [20]. Weight loss, an objective indicator of post-operative clinical status, varies widely among patients, and this may have a different impact on QoL changes after a distal gastrectomy.

Our results are consistent with those of previous studies $[21,22]$ demonstrating improved overall global health status and emotional functioning after surgery. Moreover, physical, role, and cognitive functioning decreased after surgery, whereas emotional functioning improved. Although postoperative emotional function usually improves because it is compared with QoL at the time of cancer diagnosis, as in this study, the degree of improvement may differ depending on the patient group. We suggest that patients experiencing BMI shifts after surgery may feel anxiety due to their weight loss. This is also related to the composition of the emotional functioning questionnaire items including "tense," "worry," "irritable," and "depressed." As a result, psychiatric supportive care is needed to improve functional scales, including emotional functioning, and surgeons should reassure patients that detailed medical intervention will improve their QoL after surgery.

Regarding symptom scales/items of the QLQ-C30 and QLQSTO22, this study showed that fatigue, nausea and vomiting, appetite loss, reflux symptoms, anxiety, and body image deteriorated more in patients with BMI sift to lower ranges than those with maintained BMI after distal gastrectomy. Among the scales/items, nausea and vomiting, appetite loss, and reflux symptoms were related to gastrointestinal symptoms. These symptoms are related to various factors including patient, surgical, and postoperative factors. To clarify the relationship between weight loss and gastrointestinal symptoms related to QoL, we attempted to adjust the various factors described above. Hence, patients with decreased BMI after distal gastrectomy may benefit from symptom control to improve their gastrointestinal symptoms. Anxiety and deteriorated body image need to be addressed from a psychological perspective. Anxiety and body image items in EORTC QLQ-STO22 constitute a subjective assessment of the patients' body. The questionnaire items on anxiety are similar to those of the emotional functioning questionnaire in EORTC QLQ-C30 but consist of more specific questions such as "Have you worried about your weight being too low?" In this result, the anxiety score and emotional functioning score showed conflicting results, which may indicate that the anxiety scale is highly related to the patient's weight change. Moreover, patients might regard body changes as body weight loss. As shown in this study, although some patients had a normal BMI after surgery, weight loss led to a deteriorated body image. This result might be related to fear of weight loss, which can be considered an unhealthy condition for 
patients. Thus, the surgeon should clearly inform patients that weight loss is a natural process after surgery and it is essential to assess body composition, including weight change, to reassure patients about the deterioration of their QoL.

In this study, approximately $55 \%$ of patients who underwent distal gastrectomy experienced BMI shifting after surgery and approximately $60 \%$ patients had a BMI within normal range, compared with $13 \%$ of patients with underweight BMI. To confirm QoL change in patients whose BMI shifted to underweight after surgery, these patients were compared with those who shifted to the normal range of BMI after surgery, and there was no difference in QoL change between the 2 subgroups. These study findings indicate that patients have a similar QoL when the BMI range decreases after surgery regardless of the postoperative BMI range. Therefore, it is important to analyze not only patients' QoL after surgery but also their body composition including an accurate assessment of weight change. Patients with underweight BMIs after surgery should receive intensive medical intervention to improve nutritional deficits, and should be informed that their deteriorated QoL will improve if they prevent this excess weight loss. In addition, patients with BMI shift to normal range after surgery should be informed of the accurate body composition data and be reassured that their deteriorated QoL will actually improve.

The subgroup analysis in this study suggested that the approach to weight loss in cancer patients and the healthy population should be different. In the analysis of subgroup 2 , the QoL of patients whose BMI decreased to the normal range after surgery was worse than that of patients who maintained their BMI after surgery. In the healthy population, overweight or obese people might experience a better QoL by reducing their weight. However, cancer patients may have different thoughts about weight loss compared to the healthy population. Some cancer patients may have experienced weight loss during the diagnosis of cancer and might consider their preoperative weight as healthy. These factors may cause patients QoL to deteriorate even after the postoperative BMI shifts to the normal range. Therefore, cancer patients should be managed differently from the healthy population with regard to QoL changes due to weight change. Moreover, it is important for patients to maintain their BMI after surgery and to be informed that their QoL can change due to weight loss.
The present study highlights that QoL might be affected by many factors, including preoperative patient characteristics, surgical factors, pathological factors, and postoperative patient status. These factors and the postoperative patient QoL are not simply causally related but can affect each other. Moreover, in this study, we could not clarify a causal relationship between BMI change and postoperative QoL changes. Even if this study design aimed to clarify the impact of BMI shifting on QoL after surgery, the deteriorated QoL might inversely influence the changes in BMI. Since body weight and QoL changes are significant and persistent during the first year after surgery, it is not easy to identify the causal relationship between these 2 factors at this time. However, as this study and previous study [23] noted that QoL changes were affected by many factors, BMI shifting may be just one of the factors that negatively affects QoL after surgery. In addition, as BMI is an objective index of body weight using the ratio of weight to height, we classified the patients with an emphasis on BMI shifting rather than body weight change after surgery. Further studies are warranted to clarify whether patients' QoL can be maintained by BMI or body weight control.

This study was based on the Asian population, and the distributions of BMI among Asians are distinctly left-shifted compared to those of Western populations. Thus, the results of this study should be validated for Western patients. Although the BMI range included in this study may not characterize the Western population, which has a high BMI distribution, we focused on BMI shifting to clarify changes in QoL.

In conclusion, BMI shifting after distal gastrectomy influences QoL. Focusing on the control of gastrointestinal symptoms, nutritional support, and psychological support is essential in patients with decreased BMI range after surgery. Careful followup to maintain BMI, for example by correcting dietary behavior and through intensive nutritional support, should be conducted to prevent QoL deterioration after a distal gastrectomy. Patients should be well informed about QoL changes after surgery and surgeons should reassure patients that their postoperative QoL may improve.

\section{CONFLICTS OF INTEREST}

No potential conflict of interest relevant to this article was reported.

\section{REFERENCES}

1. McCall MD, Graham PJ, Bathe OF. Quality of life: a critical outcome for all surgical treatments of gastric cancer. World J Gas- troenterol 2016;22:1101-13.

2. Yu W, Park KB, Chung HY, Kwon OK,

Lee SS. Chronological changes of quality of life in long-term survivors after gastrectomy for gastric cancer. Cancer Res Treat 2016;48:1030-6. 
3. Park KB, Lee SS, Kwon OK, Chung HY, $\mathrm{Yu}$ W. Chronological changes in quality of life after distal gastrectomy for gastric cancer. J Gastric Cancer 2017;17:110-9.

4. Davis JL, Selby LV, Chou JF, Schattner M, Ilson DH, Capanu M, et al. Patterns and predictors of weight loss after gastrectomy for cancer. Ann Surg Oncol 2016; 23:1639-45.

5. Heneghan HM, Zaborowski A, Fanning M, McHugh A, Doyle S, Moore J, et al. Prospective study of malabsorption and malnutrition after esophageal and gastric cancer surgery. Ann Surg 2015;262:803-7.

6. Bottomley A. The cancer patient and quality of life. Oncologist 2002;7:120-5.

7. Aaronson NK, Ahmedzai S, Bergman B, Bullinger M, Cull A, Duez NJ, et al. The European Organization for Research and Treatment of Cancer QLQ-C30: a qualityof-life instrument for use in international clinical trials in oncology. J Natl Cancer Inst 1993;85:365-76.

8. Vickery CW, Blazeby JM, Conroy T, Arraras J, Sezer O, Koller M, et al. Development of an EORTC disease-specific quality of life module for use in patients with gastric cancer. Eur J Cancer 2001;37:966-71.

9. Kim AR, Cho J, Hsu YJ, Choi MG, Noh JH, Sohn TS, et al. Changes of quality of life in gastric cancer patients after curative resection: a longitudinal cohort study in Korea. Ann Surg 2012;256:1008-13.

10. Karanicolas PJ, Graham D, Gonen M, Strong VE, Brennan MF, Coit DG. Quality of life after gastrectomy for adenocarci- noma: a prospective cohort study. Ann Surg 2013;257:1039-46.

11. Nishigori T, Okabe H, Tsunoda S, Shinohara H, Obama K, Hosogi H, et al. Superiority of laparoscopic proximal gastrectomy with hand-sewn esophagogastrostomy over total gastrectomy in improving postoperative body weight loss and quality of life. Surg Endosc 2017;31:3664-72.

12. Lee SS, Chung HY, Kwon OK, Yu W. Longterm quality of life after distal subtotal and total gastrectomy: symptom- and behavior-oriented consequences. Ann Surg 2016;263:738-44.

13. Park KB, Park JY, Lee SS, Kwon OK, Chung HY, Yu W. Impact of body mass index on the quality of life after total gastrectomy for gastric cancer. Cancer Res Treat 2018; 50:852-60.

14. WHO Expert Consultation. Appropriate body-mass index for Asian populations and its implications for policy and intervention strategies. Lancet 2004;363:15763.

15. Fujiwara M, Kodera Y, Kasai Y, Kanyama Y, Hibi K, Ito K, et al. Laparoscopy-assisted distal gastrectomy with systemic lymph node dissection for early gastric carcinoma: a review of 43 cases. J Am Coll Surg 2003:196:75-81.

16. Kanaya S, Gomi T, Momoi H, Tamaki N, Isobe H, Katayama T, et al. Delta-shaped anastomosis in totally laparoscopic Billroth I gastrectomy: new technique of intraabdominal gastroduodenostomy. J Am Coll Surg 2002;195:284-7.
17. Yun YH, Park YS, Lee ES, Bang SM, Heo DS, Park SY, et al. Validation of the Korean version of the EORTC QLQ-C30. Qual Life Res 2004;13:863-8.

18. Fayers PM. Interpreting quality of life data: population-based reference data for the EORTC QLQ-C30. Eur J Cancer 2001; 37:1331-4.

19. Kim YW, Baik YH, Yun YH, Nam BH, Kim DH, Choi IJ, et al. Improved quality of life outcomes after laparoscopy-assisted distal gastrectomy for early gastric cancer: results of a prospective randomized clinical trial. Ann Surg 2008;248:721-7.

20. Kiyama T, Mizutani T, Okuda T, Fujita I, Tokunaga A, Tajiri T, et al. Postoperative changes in body composition after gastrectomy. J Gastrointest Surg 2005;9:313-9.

21. Park S, Chung HY, Lee SS, Kwon O, Yu W. Serial comparisons of quality of life after distal subtotal or total gastrectomy: what are the rational approaches for quality of life management? J Gastric Cancer 2014; 14:32-8.

22. Kobayashi D, Kodera Y, Fujiwara M, Koike M, Nakayama G, Nakao A. Assessment of quality of life after gastrectomy using EORTC QLQ-C30 and STO22. World J Surg 2011;35:357-64.

23. Tanabe K, Takahashi M, Urushihara T, Nakamura Y, Yamada M, Lee SW, et al. Predictive factors for body weight loss and its impact on quality of life following gastrectomy. World J Gastroenterol 2017; 23:4823-30. 